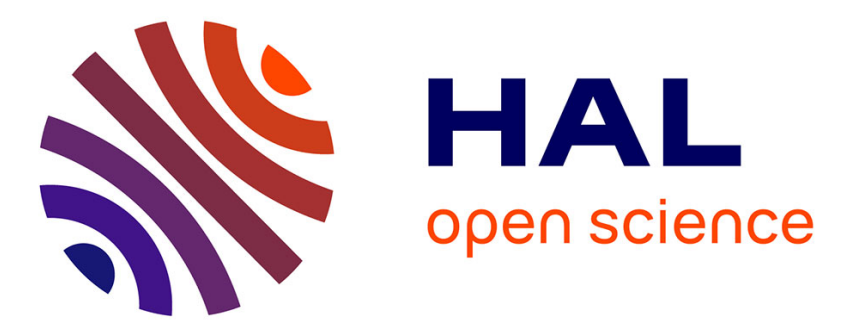

\title{
Collaborative Design Approach for the Development of an Ontology-based Decision Support System in Health Tourism
}

Daniele Spoladore, Elena Pessot, Michael Bischof, Arnulf Hartl, Marco Sacco

\section{- To cite this version:}

Daniele Spoladore, Elena Pessot, Michael Bischof, Arnulf Hartl, Marco Sacco. Collaborative Design Approach for the Development of an Ontology-based Decision Support System in Health Tourism. 22nd Working Conference on Virtual Enterprises (PRO-VE 2021), Nov 2021, Saint-Etienne, France. pp.632-639, 10.1007/978-3-030-85969-5_59 . emse-03349525

HAL Id: emse-03349525

https://hal-emse.ccsd.cnrs.fr/emse-03349525

Submitted on 25 Nov 2021

HAL is a multi-disciplinary open access archive for the deposit and dissemination of scientific research documents, whether they are published or not. The documents may come from teaching and research institutions in France or abroad, or from public or private research centers.
L'archive ouverte pluridisciplinaire HAL, est destinée au dépôt et à la diffusion de documents scientifiques de niveau recherche, publiés ou non, émanant des établissements d'enseignement et de recherche français ou étrangers, des laboratoires publics ou privés. 
Spoladore D., Pessot E., Bischof M., Hartl A., Sacco M. (2021) Collaborative Design Approach for the Development of an Ontology-Based Decision Support System in Health Tourism. In: Camarinha-Matos L.M., Boucher X., Afsarmanesh H. (eds) Smart and Sustainable Collaborative Networks 4.0. PRO-VE 2021. IFIP Advances in Information and Communication Technology, vol 629. Springer, Cham. https://doi.org/10.1007/978-3030-85969-5_59

\title{
Collaborative Design Approach for the Development of an Ontology-based Decision Support System in Health Tourism
}

\author{
Daniele Spoladore ${ }^{1,2}$, Elena Pessot ${ }^{1}$, Michael Bischof ${ }^{3}$, Arnulf $\operatorname{Hartl}^{3}$ and Marco \\ Sacco $^{1}$ \\ ${ }^{1}$ Institute of Intelligent Industrial Technologies and Systems for Advanced Manufacturing \\ (STIIMA) National Research Council of Italy \\ ${ }^{2}$ Department of Pure and Applied Sciences, Insubria University, Varese, Italy \\ ${ }^{3}$ Paracelsus Medizinische Privatuniversität, Universitätsinstitut für Ecomedicine, Salzburg, \\ Austria \\ \{daniele.spoladore, elena.pessot, marco.sacco\}@stiima.cnr.it \\ \{michael.bischof, arnulf.hartl\}@pmu.ac.at
}

\begin{abstract}
Evidence-based Health Tourism (EHT) is a branch of general tourism foreseeing tourists to travel with the aim of receiving healing treatments or enhancing a specific mental, physical or spiritual health condition through medically-proven offers. EHT competitiveness is increasingly linked to the sustainability and exploitation of unique natural resources of tourism destinations, which often lack the access to knowledge and networks of stakeholders to improve their offerings, even by the use of digital tools. This study illustrates a Collaborative Design approach for the development of an ontology-based Decision Support System for modelling the relationships between the available natural resources, the value offerings and the target groups of EHT destinations in the Alpine region. The Collaborative Design approach foresees the involvement of end-users (i.e. EHT destinations, stakeholders and tourists) as both sources of knowledge and validators of the ontology and its outputs, aiming to inform decision-making processes in a shared knowledge model.
\end{abstract}

Keywords: Health tourism, Evidence-based health tourism, Collaborative design, Ontology-based decision support system, Collaborative Ontology Engineering Methodology, Alpine region.

\section{Introduction}

Evidence-based Health Tourism (EHT) is a branch of general tourism foreseeing tourists to travel with the aim of receiving healing treatments or enhancing a specific mental, physical or spiritual health condition through medically-proven offers $[1,2]$. EHT competitiveness is increasingly linked to the sustainability and exploitation of unique natural resources of tourism destinations. Natural healing resources as waterfalls, Alpine herbs and peculiar mountain microclimates offer proven healthpromoting effects [3-5]. As central components of EHT products, they are a strong 
unique selling proposition and also increase the authenticity of these Alpine offerings. This opens up numerous approaches for involving different regional actors (e.g. tourism and medical service providers, agriculture, crafts) into the value chain [2], especially the more collaborative ones. Nevertheless, tourism destinations often lack the access to knowledge and networks of stakeholders to improve their offerings and exploit the synergic combination of possible EHT sources and activities [6]. There is the need to identify and adopt solutions that facilitate the connection among key actors by exchanging evidence-based data and systematizing knowledge and local experiences in a collaborative effort. Along with this line, several digital tools are revolutionizing healthcare sectors, including the development of solutions based on Artificial Intelligence and Semantic Web that can help cross-national interactions, redefining competitive EHT services and delivering better management strategies [7]. This work aims to develop a DSS for the creation of a shared knowledge base among EHT destinations, to support them in understanding the potentialities of their territory and to suggest them on which services and natural resource to invest [8]. Specifically, it addresses the destinations delivering healing solutions for tourists in Alpine regions, which are characterized by a high range of natural health resources and high environmental quality, but are still not sufficiently integrated in value chains to properly face the demanding market of health travelers [2]. In particular, the high demand for knowledge from different disciplines as well as the necessary involvement of numerous different actors makes it difficult for these destinations to further develop evidence- and nature-based health tourism and exploit related opportunities.

The result of this work is an ontology-based DSS that leverages the collaborative efforts of different key stakeholders of EHT as part of the HEALPS2 project, which aims at developing and improving framework conditions and tools for a better utilization of Alpine-specific natural health resources for the development of innovative tourism products and service chains. It connects academia, different business sectors such as the health sector, tourism and local service providers, as well as innovation and transfer agencies to jointly implement new business models that improve value creation across sectors in Alpine destinations. This transnational and transversal approach is built on unique Alpine natural health resources and strengthens the Alpine territorial innovation capacity. The project involves six Alpine countries (five from the EU): Austria, Italy, Germany, France, Slovenia and Switzerland, with representatives from both the academia, management bodies of mountain areas, non-governmental associations of Alpine space, thermalism and governmental Alpine networks. The development of the ontology underlying the DSS takes advantage of a collaborative design approach through the adoption of a collaborative ontology engineering methodology that involved all the stakeholders of the project.

The reminder of this work is organized as follows: Sect. 2 highlights a few studies adopting ontologies in health or medical tourism contexts, while Sect. 3 delves into the specific cooperative ontology engineering methodology and process adopted for the cooperative development of the HEALPS2 knowledge base underlying the DSS. Finally, the Conclusions summarize the main outcomes of this work. 


\section{Related Work}

The use of Semantic Web technologies - in particular, ontologies - for the development of DSSs in the tourism industry is widely documented in scientific literature. Also, the value of cooperation in ontology engineering - especially in health-related fields - is also widely established $[9,10]$. However, there are very few examples of ontologybased DSSs specifically dedicated to the fields of health and medical tourism.

Chantrapornchai et al. [11] developed an ontology dedicated to gather and organize health tourism information for the Hua Hin area in Thailand; the ontology was developed using a waterfall methodology and evaluated by eliciting domain experts opinion at the end of the development phase through questionnaires. In the field of tourist destination recommender systems, Khallouki et al. [12] adopted a domain ontology for the description of touristic context, while Frikha et al. [13] exploited ontologies to enhance user-based recommendations in the field of Tunisian medical tourism. Information regarding facilities and services in medical storage can be formalized and managed through ontologies: Lee et al. [14] proposed a smart orchestrator leveraging semantic models to formalize knowledge from the medical tourism, general tourism and medical treatment domains.

Differently from other approaches, this work introduces an ontology-based DSS able to formalize natural resources, services provided and activities based on available natural resources, stakeholders' cooperation efforts and tourism data. The ontology is developed by a methodology that foresees the involvement of various stakeholders of health tourism value chain in a cooperative effort and in multiple rounds of knowledge exchange. Thus, it relies on collaborative ontology engineering methodologies as a way to improve the correct understanding on domain knowledge in contexts characterized by many stakeholders [15], and tested in different collaborative projects $[16,17]$. In this work, the adoption of a collaborative and agile methodology is presented as a key enabler for designing (and thus developing) the DSS's ontology.

\section{Collaborative Design Approach}

This Section deepens into the collaborative approach adopted for the identification of the knowledge to be formalized, its elicitation, conceptualization and formalization with ontological languages. Through this process, informal and formal methods were conveniently adopted to ease achieving specific goals, such as identifying the most relevant bits of knowledge, produce an accessible and shared conceptual model and develop its ontological representation.

HEALPS2 research project fosters the cooperation among various stakeholders in the health tourism values chain, therefore stakeholders' involvement in the phases of knowledge elicitation and conceptualization covers a pivotal role. Stakeholders' ideas, opinions and knowledge were elicited through six national stakeholders' meetings (with the average participation of 15 stakeholders) and one international stakeholders meeting (with the participation of over 50 stakeholders). The aim of the meeting was to select the domains of interest and the relevant pieces of information for each of them. With the support of Project Partners', data collected were transformed in quantifiable 
KPIs, which were later used to provide a shared conceptualization and, finally, to formalize the ontological model. Fig. 1 sketches the process starting from stakeholders' involvement and finishing with the delivery of the ontology.

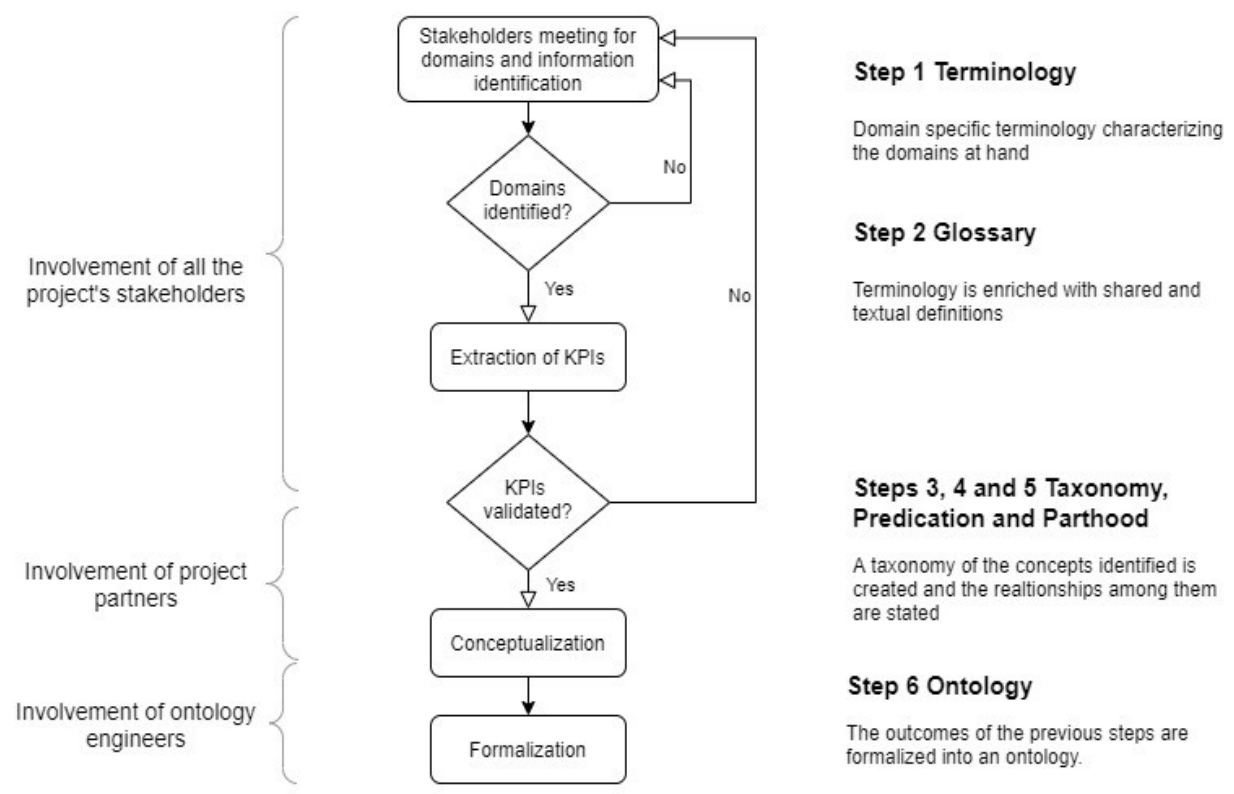

Fig. 1. The flowchart representing the collaborative approach process. On the right, the six step composing the UPONLite methodology are represented along the different phases of the process.

The ontology engineering methodology selected for the HEALPS2 project was a collaborative and agile one, namely the UPONLite methodology [18], which foresees non-experts in the field of Semantic Web to adopt common tools to provide a conceptualization of different domains of knowledge. This methodology has proven efficient in different ontology-based development processes [15]. Also, the significant number of stakeholders involved in the early phases of design, required a non-rigid structure for knowledge elicitation and subsequent conceptualization. The formalization phase - which involved mostly partners with experience in ontology development - adopted Resource Description Framework (RDF) [19], Ontology Web Language (OWL) [20] and Semantic Web Rule Language (SWRL) [21] as W3Cendorsed development languages.

\subsection{Multi-stakeholder Knowledge Elicitation Process}

The domains involved in the knowledge elicitation process were different: from a healthcare point of view, the focus was on identifying the main groups of patients who could benefit from nature-based health tourism in the Alpine space. Likewise, from a medical point of view and from a health economic and tourism perspective, it is 
important to know which natural resources can be used and how, together with the services tourism destinations can provide. Furthermore, quantitative indicators regarding the tourists' arrival, stay and provenance are also fundamental to provide a measurement of the tourism inflow in a specific area. The domains elicited by stakeholders and project partners, and for which knowledge needed to be specified were:

- A list of 19 Target Groups (TG) corresponding to some chronic impairments or physical limitations for which nature-based health tourism activity can provide benefits (e.g.: lack of mobility, diabetes and metabolic disorders, skin conditions, exhaustion and tiredness, etc.).

- Tourism in general (TiG): in this domain data regarding a specific tourism destination and its touristic inflow are detailed (tourists' arrivals, their country of origin, duration of stays, tourists' age and gender, destination population density, economic impact of tourism on the destination tourism intensity, overnight stays per 1,000 inhabitants).

- Natural Resources (NRs): this domain identifies the main natural resources in the Alpine space (blue spaces, forests, waterfalls, altitudes, protected areas, specific flora, presence of radon that can be exploited for medical purposes and presence of nature-based local products such as Alpine dairy products, farm products, honey, etc.); moreover, this domain encompasses three fundamental indicators of environmental metrics (air pollution, light pollution, noise pollution).

- Regional Features (RFs): the knowledge in this domain is descriptive, as it identifies services and characteristics a health tourist destination can have or offer (e.g.: health manager, nutritional advice, mountain hiking activities, spa treatments, physiotherapy, etc.); some services are correlated with the availability of one or more natural resources.

- Cooperation and Networking $(\mathrm{CN})$ : stakeholders recognized the role of cooperation in the success of tourism destination. In particular, they identified being part of a network (regional, national or international) as a success factor.

Together with the identification of the domains and their features, the results from the collaborative efforts of stakeholders, further informed by scientific literature, allowed to identify KPIs for each of the domain features, with the aim of making quantifiable the main features for tourist destination and their services. The consortium identified an array of data types ranging from integers for the impact that tourism destinations' features can have on a specific TG and for environmental metrics indicators, Boolean values for the existence, use and exploitation of NRs, as well as for the presence of specific services (RFs) and cooperation activities $(\mathrm{CN})$. Quantification of TiG features required different data types, including strings, integers and decimal. This step allowed to set a shared vocabulary and to specify the meaning of each terms (Steps 1 and 2 of UPONLite). 


\subsection{Agile Cooperative Methodology for Conceptualization}

Once the relevant pieces of information were identified, the conceptualization phase following the instruction from the UPONLite methodology - was conducted using conceptual maps and spreadsheets, as this kind of tools are more familiar for ontology non-experts. The result of this process further refined the elicited knowledge into a conceptual model in which each bit of information is labelled and linked to others through mathematical relationships. These relationships constitute the basis for the inference of new facts in the ontology, since they specify how a concept should change (in its classification and/or in the values it can assume) according to specific valuedriven conditions. A taxonomy of concepts (Step 3) was generated using a spreadsheet and those concepts necessary to qualify others were defined as properties (Step 4), while part-whole relationships among concepts where also formalized (Step 5).

\subsection{Development}

The conceptual model containing the mathematical relationships among concepts were translated into an ontology with the use of Protégé ontology editor [22] and using RDF and OWL to represent the concept hierarchy and SWRL to formalized relationships into rules. The HEALPS2 ontology encompasses more than 85 classes, 9 object properties, 57 datatype properties and includes 1075 individuals (for a total of 7265 axioms, including SWRL rules). Fig. 2 shows an excerpt of the developed ontology.

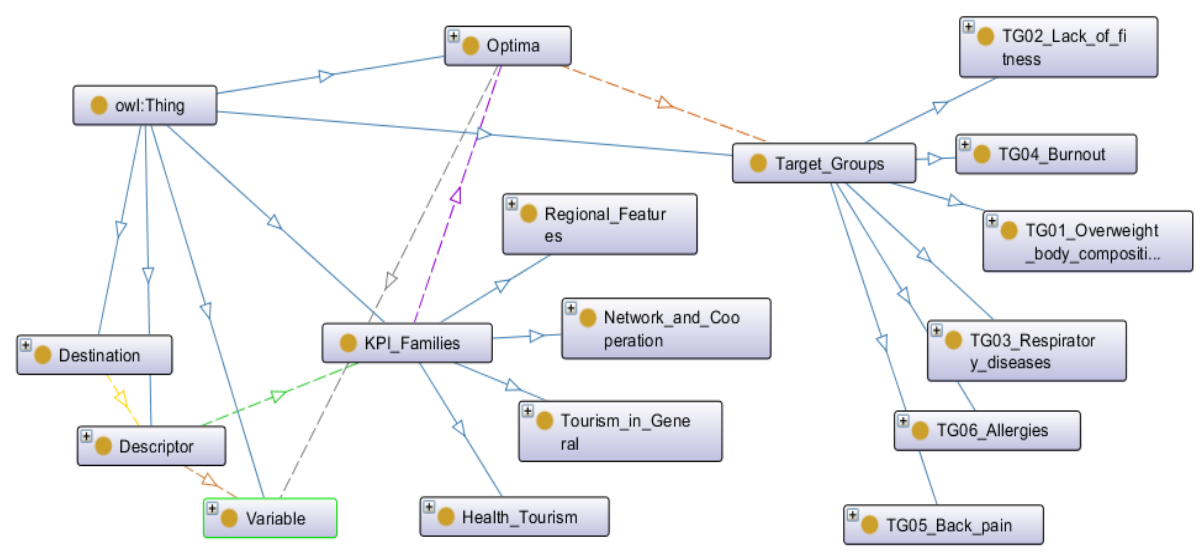

Fig. 2. A graphical representation of the main concepts represented in the HEALPS2 ontology and their relationships.

To validate the consistency of the ontology, the Pellet reasoner [23] was selected, as it is one of the few reasoners able to process SWRL built-in functions (necessary to state mathematical relationships among concepts). 


\subsection{Validation of the Ontology and Its Outputs}

The HEALPS2 methodology was presented and discussed in a second round of meetings with the key stakeholders. To populate the ontology and test the rules, stakeholders where asked to provide some information. Specifically, five national stakeholders' meetings (with the average participation of 15 stakeholders) and one international stakeholders' meeting (with the participation of over 70 stakeholders) were organized in order to collect data in input, to collect feedbacks on the base of knowledge available and to test the effectiveness of the tool. The DSS was tested with data from four Alpine destinations to check the consistency of the ontological model and the results of the reasoning process. The DSS was able to calculate the distances occurring between the predefined optimum values of KPIs and the real values of the destinations for each target group, thus contributing to identify those natural resources and/or services worth investing on. Also, in this phase the collaborative approach was judged fundamental to gain insights on the further developments of the ontology-based DSS as a reference tool for Alpine regions EHT destinations.

\section{Conclusions and Future Works}

This work developed an ontology-based DSS to support ETH destinations in innovating and enriching their product offerings based on local natural resources, and the systematic sharing of knowledge among key stakeholders to support decisions in management practices. Firstly, it contributes to the growing debates on exploiting the potentialities of digital tools in healthcare industries and the need to identify proper solutions to grow EHT industry in regional value chains. Secondly, it leverages a collaborative ontology engineering methodology to develop an evidence-based and effective ontology-based system for decision-making. In particular, the collaborative design approach reinforces the possibility of actual adoption of the DSS, and encourages the reuse of the ontology developed with stakeholders.

Future works foresee the automatic acquisition of data from stakeholders via an application and the presentation of reasoning outputs in both textual and graphical forms. Moreover, the usability of the developed tools and its outputs will be tested with stakeholders who took part in the collaborative design phase, and further users will be engaged in pilot tests with specific training modules on the developed DSS.

Acknowledgments. HEALPS2 started in October 2019 and will run until June 2022 and is co-financed by the European Regional Development Fund through the Interreg Alpine Space Programme.

\section{References}

1. Chang, L., Beise-Zee, R.: Consumer perception of healthfulness and appraisal of healthpromoting tourist destinations. Tourism Review. (2013). 
2. Steckenbauer, G.C., Tischler, S., Hartl, A., Pichler, C.: Destination and product development rested on evidence-based health tourism. The Routledge handbook of health tourism. 315-331 (2017).

3. Grafetstätter, C., Gaisberger, M., Prossegger, J., Ritter, M., Kolarž, P., Pichler, C., Thalhamer, J., Hartl, A.: Does waterfall aerosol influence mucosal immunity and chronic stress? A randomized controlled clinical trial. Journal of physiological anthropology. 36, 1-12 (2017).

4. Pieroni, A., Giusti, M.E.: Alpine ethnobotany in Italy: traditional knowledge of gastronomic and medicinal plants among the Occitans of the upper Varaita valley, Piedmont. Journal of Ethnobiology and Ethnomedicine. 5, 1-13 (2009).

5. Prossegger, J., Huber, D., Grafetstätter, C., Pichler, C., Weisböck-Erdheim, R., Iglseder, B., Wewerka, G., Hartl, A.: Effects of moderate mountain hiking and balneotherapy on community-dwelling older people: A randomized controlled trial. Experimental gerontology. 122, 74-84 (2019).

6. Pessot, E., Spoladore, D., Zangiacomi, A., Sacco, M.: Natural Resources in Health Tourism: A Systematic Literature Review. Sustainability. 13, 2661 (2021).

7. Wong, B.K.M., Hazley, S.A.S.: The future of health tourism in the industrial revolution 4.0 era. Journal of Tourism Futures. (2020).

8. Spoladore, D., Pessot, E., Sacco, M.: HEALPS 2: Tourism Based on Natural Health Resources for the Development of Alpine Regions. ERCIM News. 123, 41 (2020).

9. Spoladore, D.: Ontology-based decision support systems for health data management to support collaboration in ambient assisted living and work reintegration. In: Working Conference on Virtual Enterprises. pp. 341-352. Springer (2017).

10. Spoladore, D., Sacco, M.: Towards a Collaborative Ontology-Based Decision Support System to Foster Healthy and Tailored Diets. In: Working Conference on Virtual Enterprises. pp. 634-643. Springer (2020).

11. Chantrapornchai, C., Choksuchat, C.: Ontology construction and application in practice case study of health tourism in Thailand. SpringerPlus. 5, 1-31 (2016).

12. Khallouki, H., Abatal, A., Bahaj, M.: An ontology-based context awareness for smart tourism recommendation system. In: Proceedings of the International Conference on Learning and Optimization Algorithms: Theory and Applications. pp. 1-5 (2018).

13. Frikha, M., Mhiri, M., Gargouri, F., others: A semantic social recommender system using ontologies based approach for Tunisian tourism. (2015).

14. Lee, H.J., Park, S.Y., Jin, H.R., Sohn, M.: A smart orchestrator of ecosystem in medical tourism. In: Proceedings of the 18th Annual International Conference on Electronic Commerce: e-Commerce in Smart connected World. pp. 1-8 (2016).

15. Spoladore, D., Pessot, E.: Collaborative Ontology Engineering Methodologies for the Development of Decision Support Systems: Case Studies in the Healthcare Domain. Electronics. 10, 1060 (2021).

16. Kotis, K.I., Vouros, G.A., Spiliotopoulos, D.: Ontology engineering methodologies for the evolution of living and reused on-tologies: status, trends, findings and recommendations. The Knowledge Engineering Review. 35, (2020).

17. Simperl, E., Luczak-Rösch, M.: Collaborative ontology engineering: a survey. (2014).

18. De Nicola, A., Missikoff, M.: A lightweight methodology for rapid ontology engineering. Communications of the ACM. 59, 79-86 (2016).

19. Pan, J.Z.: Resource description framework. In: Handbook on ontologies. pp. 71-90. Springer (2009)

20. Antoniou, G., Van Harmelen, F.: Web ontology language: Owl. In: Handbook on ontologies. pp. 67-92. Springer (2004). 
21. Horrocks, I., Patel-Schneider, P.F., Boley, H., Tabet, S., Grosof, B., Dean, M., others: SWRL: A semantic web rule language combining OWL and RuleML. W3C Member submission. 21, 1-31 (2004)

22. Tudorache, T., Noy, N.F., Tu, S., Musen, M.A.: Supporting collaborative ontology development in Protégé. In: International Semantic Web Conference. pp. 17-32. Springer (2008)

23. Sirin, E., Parsia, B., Grau, B.C., Kalyanpur, A., Katz, Y.: Pellet: A practical owl-dl reasoner. Journal of Web Semantics. 5, 51-53 (2007). 